Dialoguer pour éviter les tensions

Dans l'article qui suit, Mme la Dr Morris nous permet de prendre part aux réflexions menées par une femme médecin d'un centre régional de placement et son intervention est à saluer. D'ailleurs, nous souhaitons aussi l'inverse: voir à nouveau l'Al s'intéresser davantage aux réflexions des médecins traitants. La communication n'est pas une voie à sens unique! Quand I'ORP veut déclarer I'une de nos décisions «incompréhensible», il est définitivement trop tard et on rate la chance d'utiliser toutes les ressources, justement aussi la longue expérience des médecins traitants, pour décider le meilleur pour le patient concerné.

Dans une procédure de plus en plus impersonnelle et peu juridique, la communication directe des médecins entre eux, que ce soit entre médecins de famille, spécialistes traitants, experts ou médecins de l'ORP, est restée sur le carreau au détriment du patient. Nous plaidons expressément en faveur d'une meilleure collaboration de tous les participants, justement aussi entre les services médicaux régionaux et les médecins traitants.

Dans l'avant-propos du dernier rapport de recherche publié par I'Al, Stefan Ritler, nouveau vice-directeur de l'Office fédéral des assurances sociales (OFAS) et chef de I'Al, souligne les déficits en matière d'information ainsi que les difficultés de communication et de coopération qui péjorent la réintégration des personnes malades psychiques. Nous sommes prêts à fournir notre part pour améliorer cette communication.

Dr Christine Romann, membre du Comité central de la FMH

\title{
Im Spannungsfeld verschiedener Interessen?
}

\author{
Die Aufgabe von Ärztinnen und Ärzten im Regionalen Ärztlichen Dienst (RAD) besteht \\ darin, einen geltend gemachten Gesundheitsschaden auf seine Objektivierbarkeit zu \\ überprüfen und seine Auswirkungen auf die Arbeitsfähigkeit zu beurteilen. Diese \\ Tätigkeit ist vielfältig und setzt eine grosse berufliche Erfahrung voraus. Die versiche- \\ rungsmedizinische Betrachtungsweise weist Besonderheiten auf, die juristisch vorge- \\ geben sind und die der behandelnde Arzt teilweise nicht kennt.
}

\section{Therese Morris}

Dr. med., Fachärztin für Innere Medizin FMH, arbeitet bei der IV-Stelle Bern als Ärztin im Regionalen Ärztlichen Dienst (RAD) Bern, Freibourg, Solothurn.
Korrespondenz:

Dr. med. Therese Morris

IV-Stelle Bern

Chutzenstrasse 10

CH-3001 Bern

Tel. 0313797334

therese.morris@ivbe.ch
Von 2000 bis 2005 sind die Invalidenrenten um ca. 25 Prozent auf rund 252000 gestiegen. Bei Rentenbezügern infolge psychischer Probleme lag die $\mathrm{Zu}$ wachsrate sogar bei 50 Prozent. Mit der 4. IV-Revision im Jahr 2004 wurden die RAD eingeführt. Seither ging die Zahl der Rentenbezüge leicht zurück. Der Rentenanstieg bei Versicherten mit einer psychischen Beeinträchtigung wurde deutlich gebremst. Besteht ein Zusammenhang zwischen der Einführung der RAD und dem Rückgang von Neu-Renten? Werden RAD-Ärzte dazu missbraucht, geschuldete Leistungen zu verhindern?

RAD-Ärzte überprüfen einen geltend gemachten Gesundheitsschaden auf seine Objektivierbarkeit. Vor allem beurteilen sie die Auswirkungen auf die Arbeitsfähigkeit in der bisherigen oder in einer angepassten Tätigkeit. Es liegt in ihrem Ermessen zu entscheiden, ob die vorhandenen Arztberichte für eine fundierte Stellungnahme genügen, ob eine eigene Untersuchung des Versicherten notwendig ist oder ob interne oder externe Fachspezialisten hinzuzuziehen sind.

\section{Interdisziplinäre Zusammenarbeit}

Am Entscheidungsprozess sind mehrere Abteilungen der IV-Stelle beteiligt, beispielsweise Fallmanage- ment, Eingliederungsmanagement, Abklärungsdienst, Rechtsdienst und RAD. Nach dem Grundsatz «Eingliederung vor Rente» ist die Zusammenarbeit zwischen Eingliederungsfachperson und RAD-Arzt besonders wichtig. In unklaren Fällen zeigt er der Eingliederungsfachperson anhand des vorliegenden Krankheitsbildes auf, welche Funktionen bei der betroffenen Person erhalten sind und welche Tätigkeiten dieser zugemutet werden können. Zu diesem «Zumutbarkeitsprofil» gehören Angaben zur Art der Tätigkeit wie Körperposition, Gewichtsbelastung, Stressbelastung, Lufthygiene, intellektuelle Ansprüche usw., sowie Angaben zum zeitlichen Pensum und zu der zu erwartenden Leistungsfähigkeit während der Arbeit. Wenn eine versicherte Person ihre angestammte Tätigkeit nicht mehr weiterführen kann und Alternativmöglichkeiten nicht klar gegeben sind, steht der IV-Stelle Bern mit der arbeitsmarktlich-medizinischen Abklärung (AMA) ein spezielles Abklärungsinstrument zur Verfügung. In einem vier Wochen dauernden Programm werden verschiedene Fähigkeiten wie Feinmotorik, handwerkliches Geschick, aber auch planerische Begabung oder die Eignung für Computerarbeit im Hinblick auf eine mögliche Verweistätigkeit ge- 
testet. Zugleich wird die Motivation beurteilt, ohne die eine Wiedereingliederung in den Arbeitsprozess zum Scheitern verurteilt ist. Während der ganzen AMA-Abklärung wird der Versicherte von einer Eingliederungsfachperson, einem RAD-Arzt sowie einem Arbeitsagogen der entsprechenden Institution begleitet.

\section{Versicherungsmedizinische Besonderheiten}

Die versicherungsmedizinische Betrachtungsart weist juristisch vorgegebene Besonderheiten auf, weshalb Entscheide der IV und damit auch des RAD für Aussenstehende oft nicht verständlich sind. Ein weitgehend akzeptiertes Beispiel ist das reine Suchtgeschehen. Die IV geht davon aus, dass nach der Überwindung der Sucht eine Arbeitsfähigkeit besteht, und mutet der betroffenen Person eine Entzugsbehandlung zu, auch wenn diese oft nicht erfolgreich ist. Weniger akzeptiert ist das Beispiel der chronischen Schmerzkrankheit. Ein Schmerzgeschehen, das zur Arbeitsunfähigkeit führt, muss eine objektivierbare somatische Grundlage haben. Ansonsten ist es gemäss geltendem Recht in der Regel nicht versichert. Hier besteht ein grosses Konfliktpotenzial mit dem behandelnden Arzt. Dieser sieht einen glaubhaft leidenden Menschen, der sich oft auch in einer ausgeprägten psychosozialen Belastungssituation befindet. Für ihn ist es klar, dass seinem Patienten geholfen werden muss, unter anderem auch in Form einer finanziellen Existenzsicherung wie der IV-Rente. Dabei wird oft ausser acht gelassen, dass der «Kampf für eine IV-Rente» nicht unbedingt die bestmögliche Hilfe darstellt. Noch verstehen die meisten Menschen die Arbeit nicht als notwendiges Übel, sondern als sinnvollen Beitrag für die Gesellschaft, mit der man Anerkennung findet und durch die das soziale Leben

\footnotetext{
Dans un champ de tensions entre intérêts divergents?

Les nouvelles rentes ont diminué depuis l'introduction des Services médicaux régionaux (SMR) en 2004. Existe-t-il un lien entre les SMR et cette diminution? Les médecins des SMR ont-ils le devoir d'empêcher des prestations d'assurance? Plusieurs spécialistes de l'office $\mathbf{A l}$ - personnes spécialisées en assurance ou en réinsertion professionnelle, juristes et médecins - participent au processus décisionnel. Conformément au principe de la «réinsertion avant la rente», les personnes chargées de la réinsertion et les médecins des SMR travaillent en étroite collaboration. Par exemple, le profil établi par un médecin concernant ce que l'on peut raisonnablement exiger du patient constitue la base indiquant les activités que l'assuré peut exercer et à quel taux d'engagement, compte tenu de son état de santé.

Le travail des médecins SMR est exigeant, varié et toujours passionnant. Ces médecins se trouvent néanmoins aussi dans un champ de tensions entre des intérêts divergents. D'un côté, ils voient la personne concernée, laquelle est diminuée dans sa capacité à travailler en raison de son état de santé et aimerait toucher des prestations de I'Al. D'un autre côté, ils sont conscients de leur responsabilité face à la société. Leur exigence de ne promettre des prestations qu'en cas de droit prouvé est absolument justifiée. Dans son article, Therese Morris présente le travail des SMR, explique les particularités de la médecine des assurances et répond aux questions posées en entrée.
}

strukturiert wird.

$\mathrm{Zu}$ Missverständnissen kann auch die Definition des Begriffs «Gesundheit» führen. Während die WHO und damit auch die behandelnde Ärzteschaft Gesundheit als körperliches, seelisches und soziales Wohlbefinden definiert, anerkennt die IV die soziale Komponente nicht.

\section{Voraussetzungen für Leistungen der IV}

Grundvoraussetzung für Leistungen ist der versicherte Gesundheitsschaden, wobei die Diagnose einer anerkannten ICD-10-Codierung entsprechen muss. Der RAD-Arzt überprüft die korrekte Diagnosestellung. Differenzen ergeben sich häufig im psychiatrischen Bereich, beispielsweise bei der Beurteilung des Schweregrades einer Depression oder der in der letzten Zeit inflationär gestellten Diagnose einer posttraumatischen Belastungsstörung. Nur aufgrund einer bestimmten Diagnose kann die Frage nach dem Anrecht auf Versicherungsleistungen nicht beantwortet werden. Vielmehr geht es darum, den Schweregrad der Erkrankung und deren Auswirkungen auf die Berufsausübung zu evaluieren. Dazu gehören Kenntnisse über die bisherige Tätigkeit des Versicherten und die Beurteilung möglicher Alternativtätigkeiten. Ferner muss sich der RAD-Arzt mit der versicherungsmedizinischen Betrachtungsweise auseinandersetzen und auch juristische Einwände bearbeiten. In diesem komplexen Prozess kann es zu Fehleinschätzungen kommen. Entscheide, die auf unkorrekten Beurteilungen beruhen, sollen mit den betroffenen Parteien verhandelt werden, notfalls vor Gericht.

Mit Inkrafttreten der 5. IV-Revision wurde die Bekämpfung des Versicherungsmissbrauchs neu ausgerichtet. Seither können - wenn alle anderen Möglichkeiten zur Ermittlung des Sachverhalts ausgeschöpft sind - Observationen in die Wege geleitet werden. Es obliegt dem RAD-Arzt, Videos von observierten Personen aus medizinischer Sicht zu kommentieren. Auch wenn dieses Thema in der Politik aktuell heiss diskutiert wird, handelt es sich um Einzelfälle.

\section{Fazit}

Die Titelfrage kann ich klar mit «Ja» beantworten. Auf der einen Seite befindet sich die versicherte Person, die bei objektivierter Beeinträchtigung ihrer Leistungsfähigkeit aufgrund eines Gesundheitsschadens Anrecht auf Leistungen der IV hat. Dass ein solcher Leistungsanspruch nicht von allen Parteien gleich beurteilt wird, liegt in der Natur der Sache. Auf der anderen Seite steht die Verantwortung gegenüber der Gesellschaft. Ihre Forderung, dass die gemeinschaftlichen Gelder nur bei ausgewiesenem Anspruch zugesprochen werden, ist legitim.

Die Arbeit der RAD-Ärztinnen und -Ärzte ist anspruchsvoll, vielseitig und spannend. Sie setzt eine grosse berufliche Erfahrung und ein - richtig verstandenes - soziales Engagement voraus. Aufgabe des RAD ist auch, behandelnde Ärzte einzubeziehen und mit ihnen den Dialog zu suchen. 